\title{
A multiple Hilbert-type integral inequality with a non-homogeneous kernel
}

\author{
Qiliang Huang* and Bicheng Yang
}

"Correspondence: qlhuang@yeah.net Department of Mathematics, Guangdong University of

Education, Guangzhou, Guangdong 510303, P.R. China

\begin{abstract}
By using the way of weight functions and the technic of real analysis, a multiple Hilbert-type integral inequality with a non-homogeneous kernel is given. The operator expression with the norm, the reverses and some examples with the particular kernels are also considered.
\end{abstract}

MSC: 47A07; 26D15

Keywords: multiple Hilbert-type integral inequality; kernel; weight function; norm; operator

\section{Introduction}

If $p>1, \frac{1}{p}+\frac{1}{q}=1, f(\geq 0) \in L^{p}(0, \infty), g(\geq 0) \in L^{q}(0, \infty),\|f\|_{p},\|g\|_{q}>0$, then we have the following equivalent inequalities (cf. [1]):

$$
\begin{aligned}
& \int_{0}^{\infty} \int_{0}^{\infty} \frac{f(x) g(y)}{x+y} d x d y<\frac{\pi}{\sin (\pi / p)}\|f\|_{p}\|g\|_{q}, \\
& \left\{\int_{0}^{\infty}\left(\int_{0}^{\infty} \frac{f(x)}{x+y} d x\right)^{p} d y\right\}^{\frac{1}{p}}<\frac{\pi}{\sin (\pi / p)}\|f\|_{p},
\end{aligned}
$$

where the constant factor $\frac{\pi}{\sin (\pi / p)}$ is the best possible. (1) is the well-known Hardy-Hilbert integral inequality. Define the Hardy-Hilbert integral operator $T: L^{p}(0, \infty) \rightarrow L^{p}(0, \infty)$ as follows: for $f \in L^{p}(0, \infty)$, Tf $(y):=\int_{0}^{\infty} \frac{1}{x+y} f(x) d x(y \in(0, \infty))$.

Then in view of (2), it follows $\|T f\|_{p}<\frac{\pi}{\sin (\pi / p)}\|f\|_{p}$ and $\|T\| \leq \frac{\pi}{\sin (\pi / p)}$. Since the constant is the best possible, we find $\|T\|=\frac{\pi}{\sin (\pi / p)}$.

Inequalities (1) and (2) and the operator are important in analysis and its applications (cf. $[2,3])$. In 2002, [4] considered the property of the Hardy-Hilbert integral operator and gave an improvement of (1) (for $p=q=2$ ). In 2004, by introducing another pair of conjugate exponents $(r, s)\left(r>1, \frac{1}{r}+\frac{1}{s}=1\right)$ and an independent parameter $\lambda>0$, [5] gave the best extensions of (1) as follows:

$$
\int_{0}^{\infty} \int_{0}^{\infty} \frac{f(x) g(y)}{x^{\lambda}+y^{\lambda}} d x d y<\frac{\pi}{\lambda \sin (\pi / r)}\|f\|_{p, \phi}\|g\|_{q, \psi}
$$

where $\phi(x)=x^{p\left(1-\frac{\lambda}{r}\right)-1}, \psi(x)=x^{q\left(1-\frac{\lambda}{s}\right)-1},\|f\|_{p, \phi}=\left\{\int_{0}^{\infty} \phi(x) f^{p}(x) d x\right\}^{\frac{1}{p}}>0,\|g\|_{q, \psi}>0$. In 2007, [6] gave the following inequality with the best constant $B\left(\frac{\lambda}{2}, \frac{\lambda}{2}\right)(\lambda>0 ; B(u, v)$ is

\section{Springer}

(c) 2013 Huang and Yang; licensee Springer. This is an Open Access article distributed under the terms of the Creative Commons Attribution License (http://creativecommons.org/licenses/by/2.0), which permits unrestricted use, distribution, and reproduction in any medium, provided the original work is properly cited. 
the beta function):

$$
\int_{0}^{\infty} \int_{0}^{\infty} \frac{f(x) g(y)}{(1+x y)^{\lambda}} d x d y<B\left(\frac{\lambda}{2}, \frac{\lambda}{2}\right)\left\{\int_{0}^{\infty} x^{1-\lambda} f^{2}(x) d x \int_{0}^{\infty} x^{1-\lambda} g^{2}(x) d x\right\}^{\frac{1}{2}} .
$$

In 2009, [7] gave an extension of (4) in $\mathbf{R}^{2}$ with the kernel $\frac{1}{|1+x y|^{\lambda}}(0<\lambda<1)$; [8] gave another extension of (4) to the general kernel $k_{\lambda}(1, x y)(\lambda>0)$ with a pair of conjugate exponents $(p, q)$ and obtained the following multiple Hilbert-type integral inequality. Suppose that $n \in \mathbf{N} \backslash\{1\}, p_{i}>1, \sum_{i=1}^{n} \frac{1}{p_{i}}=1, \lambda>0, k_{\lambda}\left(x_{1}, \ldots, x_{n}\right) \geq 0$ is a measurable function of $-\lambda$-degree in $\mathbf{R}_{+}^{n}$, and for any $\left(r_{1}, \ldots, r_{n}\right)\left(r_{i}>1\right)$, satisfies $\sum_{i=1}^{n} \frac{1}{r_{i}}=1$ and

$$
k_{\lambda}=\int_{\mathbf{R}_{+}^{n-1}} k_{\lambda}\left(u_{1}, \ldots, u_{n-1}, 1\right) \prod_{j=1}^{n-1} u_{j}^{\frac{\lambda}{r_{j}}-1} d u_{1} \cdots d u_{n-1}>0 .
$$

If $\phi_{i}(x)=x^{p_{i}\left(1-\frac{\lambda}{r_{i}}\right)-1}, f_{i}(\geq 0) \in L_{\phi_{i}}^{p_{i}}(0, \infty),\|f\|_{p_{i, \phi_{i}}}>0(i=1, \ldots, n)$, then we have the following inequality:

$$
\int_{\mathbf{R}_{+}^{n}} k_{\lambda}\left(x_{1}, \ldots, x_{n}\right) \prod_{i=1}^{n} f_{i}\left(x_{i}\right) d x_{1} \cdots d x_{n}<k_{\lambda} \prod_{i=1}^{n}\left\|f_{i}\right\|_{p_{i}, \phi_{i}},
$$

where the constant factor $k_{\lambda}$ is the best possible. For $n=2, k_{\lambda}(x, y)=\frac{1}{x^{\lambda}+y^{\lambda}}$ in (5), we obtain (3). Inequality (5) is an extension of the results in [9-12] and [13]. In recent years, [14] and [15] considered some Hilbert-type operators relating (1)-(3); [16] also considered a multiple Hilbert-type integral operator with the homogeneous kernel of $-n+1$-degree and the relating particular case of (5) (for $\lambda=n-1, \frac{1}{r_{i}}=\frac{1}{n-1}\left(1-\frac{1}{p_{i}}\right)$ ).

In this paper, by using the way of weight functions and the technic of real analysis, a multiple Hilbert-type integral inequality with a non-homogeneous kernel is given. The operator expression with the norm, the reverses and some examples with the particular kernels are considered.

\section{Some lemmas}

Lemma 1 If $n \in \mathbf{N} \backslash\{1\}, \lambda_{i} \in \mathbf{R}(i=1, \ldots, n), \sum_{i=1}^{n} \frac{1}{p_{i}}=1$, then we have

$$
A:=\prod_{i=1}^{n}\left[x_{i}^{\left(\lambda_{i}-1\right)\left(1-p_{i}\right)} \prod_{j=1(j \neq i)}^{n} x_{j}^{\lambda_{j}-1}\right]^{\frac{1}{p_{i}}}=1 .
$$

Proof We find

$$
\begin{aligned}
A & :=\prod_{i=1}^{n}\left[x_{i}^{\left(\lambda_{i}-1\right)\left(1-p_{i}\right)+1-\lambda_{i}} \prod_{j=1}^{n} x_{j}^{\lambda_{j}-1}\right]^{\frac{1}{p_{i}}} \\
& =\prod_{i=1}^{n}\left[x_{i}^{\left(1-\lambda_{i}\right) p_{i}} \prod_{j=1}^{n} x_{j}^{\lambda_{j}-1}\right]^{\frac{1}{p_{i}}}=\prod_{i=1}^{n} x_{i}^{1-\lambda_{i}}\left(\prod_{j=1}^{n} x_{j}^{\lambda_{j}-1}\right)^{\sum_{i=1}^{n} \frac{1}{p_{i}}},
\end{aligned}
$$

and then (6) is valid. 
Definition 1 If $n \in \mathbf{N}, \mathbf{R}_{+}^{n}:=\left\{\left(x_{1}, \ldots, x_{n}\right) \mid x_{i}>0(i=1, \ldots, n)\right\}, \lambda \in \mathbf{R}, k_{\lambda}\left(x_{1}, \ldots, x_{n}\right)$ is a measurable function in $\mathbf{R}_{+}^{n}$ such that for any $u>0$ and $\left(x_{1}, \ldots, x_{n}\right) \in \mathbf{R}_{+}^{n}, k_{\lambda}\left(u x_{1}, \ldots, u x_{n}\right)=$ $u^{-\lambda} k_{\lambda}\left(x_{1}, \ldots, x_{n}\right)$, then we call $k_{\lambda}\left(x_{1}, \ldots, x_{n}\right)$ the homogeneous function of $-\lambda$-degree in $\mathbf{R}_{+}^{n}$.

Lemma 2 Suppose $n \in \mathbf{N} \backslash\{1\}, \lambda_{i} \in \mathbf{R}(i=1, \ldots, n), \lambda_{n}=\sum_{i=1}^{n-1} \lambda_{i}=\frac{\lambda}{2}, k_{\lambda}\left(x_{1}, \ldots, x_{n}\right) \geq 0$ is a homogeneous function of $-\lambda$-degree. If

$$
\begin{aligned}
H(i):= & \int_{\mathbf{R}_{+}^{n-1}} k_{\lambda}\left(u_{1}, \ldots, u_{i-1}, 1, u_{i+1}, \ldots, u_{n}\right) \\
& \times \prod_{j=1(j \neq i)}^{n} u_{j}^{\lambda_{j}-1} d u_{1} \cdots d u_{i-1} d u_{i+1} \cdots d u_{n} \quad(i=1, \ldots, n)
\end{aligned}
$$

satisfying $k_{\lambda}:=H(n) \in \mathbf{R}$, then each $H(i)=H(n)=k_{\lambda}$ and for any $i=1, \ldots, n$,

$$
\begin{aligned}
\omega_{i}\left(x_{i}\right):= & x_{i}^{\lambda_{i}} \int_{\mathbf{R}_{+}^{n-1}} k_{\lambda}\left(x_{1} x_{n}, \ldots, x_{n-1} x_{n}, 1\right) \\
& \times \prod_{j=1(j \neq i)}^{n} x_{j}^{\lambda_{j}-1} d x_{1} \cdots d x_{i-1} d x_{i+1} \cdots d x_{n}=k_{\lambda} \quad(\in \mathbf{R}) .
\end{aligned}
$$

Proof Setting $u_{j}=u_{n} v_{j}(j \neq i, n)$ in the integral $H(i)$, we find

$$
\begin{aligned}
H(i)= & \int_{\mathbf{R}_{+}^{n-1}} k_{\lambda}\left(v_{1}, \ldots, v_{i-1}, u_{n}^{-1}, v_{i+1}, \ldots, v_{n-1}, 1\right) \prod_{j=1(j \neq i)}^{n-1} v_{j}^{\lambda_{j}-1} \\
& \times u_{n}^{-1-\lambda_{i}} d v_{1} \cdots d v_{i-1} d v_{i+1} \cdots d v_{n-1} d u_{n} .
\end{aligned}
$$

Setting $v_{i}=u_{n}^{-1}$ in the above integral, we obtain $H(i)=H(n)$. Setting $x_{n}^{\prime}=x_{n}^{-1}$ in (7), since $\lambda-\lambda_{n}=\lambda_{n}$, we find

$$
\begin{aligned}
\omega_{i}\left(x_{i}\right)= & x_{i}^{\lambda_{i}} \int_{\mathbf{R}_{+}^{n-1}} k_{\lambda}\left(x_{1}, \ldots, x_{n-1}, x_{n}^{-1}\right) x_{n}^{-\lambda_{n}-1} \\
& \times \prod_{j=1(j \neq i)}^{n-1} x_{j}^{\lambda_{j}-1} d x_{1} \cdots d x_{i-1} d x_{i+1} \cdots d x_{n} \\
= & x_{i}^{\lambda_{i}} \int_{\mathbf{R}_{+}^{n-1}} k_{\lambda}\left(x_{1}, \ldots, x_{n-1}, x_{n}^{\prime}\right)\left(x_{n}^{\prime}\right)^{\lambda_{n}+1} \\
& \times \prod_{j=1(j \neq i)}^{n-1} x_{j}^{\lambda_{j}-1} d x_{1} \cdots d x_{i-1} d x_{i+1} \cdots d x_{n-1}\left(x_{n}^{\prime}\right)^{-2} d x_{n}^{\prime} \\
= & x_{i}^{\lambda_{i}} \int_{\mathbf{R}_{+}^{n-1}} k_{\lambda}\left(x_{1}, \ldots, x_{n-1}, x_{n}^{\prime}\right)\left(x_{n}^{\prime}\right)^{\lambda_{n}-1} \\
& \times \prod_{j=1(j \neq i)}^{n-1} x_{j}^{\lambda_{j}-1} d x_{1} \cdots d x_{i-1} d x_{i+1} \cdots d x_{n-1} d x_{n}^{\prime} .
\end{aligned}
$$

Setting $u_{j}=x_{j} / x_{i}(j \neq i, n)$ and $u_{n}=x_{n}^{\prime} / x_{i}$ in the above integral, we find $\omega_{i}\left(x_{i}\right)=H(i)=$ $H(n)=k_{\lambda}$. 
Lemma 3 With the assumptions given in Lemma 2, then

$$
k\left(\widetilde{\lambda}_{1}, \ldots, \tilde{\lambda}_{n-1}\right):=\int_{\mathbf{R}_{+}^{n-1}} k_{\lambda}\left(u_{1}, \ldots, u_{n-1}, 1\right) \prod_{j=1}^{n-1}{\tilde{\lambda_{j}}}^{\tilde{j}_{j}-1} d u_{1} \cdots d u_{n-1}
$$

is finite in a neighborhood of $\left(\lambda_{1}, \ldots, \lambda_{n-1}\right)$ if any only if $k\left(\widetilde{\lambda}_{1}, \ldots, \widetilde{\lambda}_{n-1}\right)$ is continuous at $\left(\lambda_{1}, \ldots, \lambda_{n-1}\right)$.

Proof The sufficiency property is obvious. We prove the necessary property of the condition by mathematical induction in the following. For $n=2$, there exists $I:=\left\{\widetilde{\lambda}_{1} \mid \widetilde{\lambda}_{1}=\right.$ $\left.\lambda_{1}+\delta_{1},\left|\delta_{1}\right| \leq \delta_{0}, \delta_{0}>0\right\}$ such that for any $\tilde{\lambda}_{1} \in I, k\left(\widetilde{\lambda}_{1}\right) \in \mathbf{R}$. Since for $\tilde{\lambda}_{1}=\lambda_{1}+\delta_{1} \in I\left(\delta_{1} \neq 0\right)$,

$$
\begin{aligned}
& k\left(\lambda_{1}+\delta_{1}\right)=\int_{0}^{1} k_{\lambda}\left(u_{1}, 1\right) u_{1}^{\lambda_{1}+\delta_{1}-1} d u_{1}+\int_{1}^{\infty} k_{\lambda}\left(u_{1}, 1\right) u_{1}^{\lambda_{1}+\delta_{1}-1} d u_{1}, \\
& k_{\lambda}\left(u_{1}, 1\right) u_{1}^{\lambda_{1}+\delta_{1}-1} \leq k_{\lambda}\left(u_{1}, 1\right) u_{1}^{\lambda_{1}-\delta_{0}-1} d u_{1}, \quad u_{1} \in(0,1] \\
& k_{\lambda}\left(u_{1}, 1\right) u_{1}^{\lambda_{1}+\delta_{1}-1} \leq k_{\lambda}\left(u_{1}, 1\right) u_{1}^{\lambda_{1}+\delta_{0}-1} d u_{1}, \quad u_{1} \in(1, \infty),
\end{aligned}
$$

and $k\left(\lambda_{1}-\delta_{0}\right)+k\left(\lambda_{1}+\delta_{0}\right)<\infty$, then by the Lebesgue control convergence theorem (cf. [17]), it follows $k\left(\lambda_{1}+\delta_{1}\right)=k\left(\lambda_{1}\right)+o(1)\left(\delta_{1} \rightarrow 0\right)$. Assuming that for $n(\geq 2), k\left(\widetilde{\lambda}_{1}, \ldots, \widetilde{\lambda}_{n-1}\right)$ is continuous at $\left(\lambda_{1}, \ldots, \lambda_{n-1}\right)$, then for $n+1$, by the result of $n=2$, since $k\left(\lambda_{1}+\delta_{1}, \ldots, \lambda_{n}+\delta_{n}\right)$ is finite in a neighborhood of $\left(\lambda_{1}, \ldots, \lambda_{n}\right)$, we find

$$
\begin{aligned}
& \lim _{\delta_{n} \rightarrow 0} k\left(\lambda_{1}+\delta_{1}, \ldots, \lambda_{n}+\delta_{n}\right) \\
& \quad=\lim _{\delta_{n} \rightarrow 0} \int_{0}^{\infty}\left(\int_{\mathbf{R}_{+}^{n-1}} k_{\lambda}\left(u_{1}, \ldots, u_{n}, 1\right) \prod_{j=1}^{n-1} u_{j}^{\lambda_{j}+\delta_{j}-1} d u_{1} \cdots d u_{n-1}\right) u_{n}^{\lambda_{n}+\delta_{n}-1} d u_{n} \\
& \quad=\int_{0}^{\infty}\left(\int_{\mathbf{R}_{+}^{n-1}} k_{\lambda}\left(u_{1}, \ldots, u_{n}, 1\right) \prod_{j=1}^{n-1} u_{j}^{\lambda_{j}+\delta_{j}-1} d u_{1} \cdots d u_{n-1}\right) u_{n}^{\lambda_{n}-1} d u_{n} \\
& =\int_{\mathbf{R}_{+}^{n-1}}\left(\int_{0}^{\infty} k_{\lambda}\left(u_{1}, \ldots, u_{n}, 1\right) u_{n}^{\lambda_{n}-1} d u_{n}\right) \prod_{j=1}^{n-1} u_{j}^{\lambda_{j}+\delta_{j}-1} d u_{1} \cdots d u_{n-1},
\end{aligned}
$$

then by the assumption for $n$, it follows

$$
\lim _{\delta_{n} \rightarrow 0} k\left(\lambda_{1}+\delta_{1}, \ldots, \lambda_{n}+\delta_{n}\right)=k\left(\lambda_{1}, \ldots, \lambda_{n}\right)+o(1) \quad\left(\delta_{i} \rightarrow 0, i=1, \ldots, n-1\right) .
$$

By mathematical induction, we prove that for $n \in \mathbf{N} \backslash\{1\}, k\left(\tilde{\lambda}_{1}, \ldots, \tilde{\lambda}_{n-1}\right)$ is continuous at $\left(\lambda_{1}, \ldots, \lambda_{n-1}\right)$.

Lemma 4 With the assumptions given in Lemma 2, if there exists $\delta>0$ such that for $\max _{1 \leq i \leq n-1}\left\{\left|\delta_{i}\right|\right\}<\delta, k\left(\lambda_{1}+\delta_{1}, \ldots, \lambda_{n-1}+\delta_{n-1}\right) \in \mathbf{R}, p_{i} \in \mathbf{R} \backslash\{0,1\}(i=1, \ldots, n), 0<\varepsilon<$ $\min _{1 \leq i \leq n}\left\{\left|p_{i}\right|\right\} \delta$, then we have

$$
\begin{aligned}
I_{\varepsilon}:= & \varepsilon \int_{1}^{\infty} \cdots \int_{1}^{\infty}\left[\int_{0}^{1} x_{n}^{\lambda_{n}+\frac{\varepsilon}{p_{n}}-1} k_{\lambda}\left(x_{1} x_{n}, \ldots, x_{n-1} x_{n}, 1\right) d x_{n}\right] \\
& \times \prod_{j=1}^{n-1} x_{j}^{\lambda_{j}-\frac{\varepsilon}{p_{j}}-1} d x_{1} \cdots d x_{n-1}=k_{\lambda}+o(1) \quad\left(\varepsilon \rightarrow 0^{+}\right) .
\end{aligned}
$$


Proof Setting $x_{n}^{\prime}=x_{n}^{-1}$ in (8), we find

$$
\begin{aligned}
I_{\varepsilon}:= & \varepsilon \int_{1}^{\infty} \ldots \int_{1}^{\infty}\left[\int_{1}^{\infty}\left(x_{n}^{\prime}\right)^{-\lambda_{n}-\frac{\varepsilon}{p_{n}}-1} k_{\lambda}\left(\frac{x_{1}}{x_{n}^{\prime}}, \ldots, \frac{x_{1}}{x_{n}^{\prime}}, 1\right) d x_{n}^{\prime}\right] \\
& \times \prod_{j=1}^{n-1} x_{j}^{\lambda_{j}-\frac{\varepsilon}{p_{j}}-1} d x_{1} \cdots d x_{n-1}=k_{\lambda}+o(1) .
\end{aligned}
$$

Setting $u_{j}=x_{j} / x_{n}^{\prime}(j=1, \ldots, n-1)$ in the above integral, since $\lambda-\lambda_{n}=\lambda_{n}$, we find (replacing $x_{n}^{\prime}$ by $\left.x_{n}\right)$

$$
I_{\varepsilon}=\varepsilon \int_{1}^{\infty} x_{n}^{-1-\varepsilon}\left[\int_{x_{n}^{-1}}^{\infty} \cdots \int_{x_{n}^{-1}}^{\infty} k_{\lambda}\left(u_{1}, \ldots, u_{n-1}, 1\right) \prod_{j=1}^{n-1} u_{j}^{\lambda_{j}-\frac{\varepsilon}{p_{j}}-1} d u_{1} \cdots d u_{n-1}\right] d x_{n} .
$$

Setting $D_{j}:=\left\{\left(u_{1}, \ldots, u_{n-1}\right) \mid u_{j} \in\left(0, x_{n}^{-1}\right), u_{k} \in(0, \infty)(k \neq j)\right\}$ and

$$
A_{j}\left(x_{n}\right):=\int \cdots \int_{D_{j}} k_{\lambda}\left(u_{1}, \ldots, u_{n-1}, 1\right) \prod_{j=1}^{n-1} u_{j}^{\lambda_{j}-\frac{\varepsilon}{p_{j}}-1} d u_{1} \cdots d u_{n-1},
$$

by (9), it follows

$$
I_{\varepsilon} \geq \int_{\mathbf{R}_{+}^{n-1}} k_{\lambda}\left(u_{1}, \ldots, u_{n-1}, 1\right) \prod_{j=1}^{n-1} u_{j}^{\lambda_{j}-\frac{\varepsilon}{p_{j}}-1} d u_{1} \cdots d u_{n-1}-\varepsilon \sum_{j=1}^{n-1} \int_{1}^{\infty} x_{n}^{-1} A_{j}\left(x_{n}\right) d x_{n} .
$$

Without loss of generality, we estimate the case of $j=n$, e.t.

$\int_{1}^{\infty} x_{n}^{-1} A_{n-1}\left(x_{n}\right) d x_{n}=O(1)$. In fact, setting $\alpha>0$, such that $\left|\frac{\varepsilon}{p_{n-1}}+\alpha\right|<\delta$, since $-u_{n-1}^{\alpha} \times$ $\ln u_{n-1} \rightarrow 0\left(u_{n-1} \rightarrow 0^{+}\right)$, there exists $M>0$ such that $-u_{n-1}^{\alpha} \ln u_{n-1} \leq M\left(u_{n-1} \in(0,1]\right)$, and then by the Fubini theorem, it follows

$$
\begin{aligned}
0 & \leq \int_{1}^{\infty} x_{n}^{-1} A_{n-1}\left(x_{n}\right) d x_{n} \\
& =\int_{1}^{\infty} x_{n}^{-1}\left[\int_{\mathbf{R}_{+}^{n-2}} \int_{0}^{x_{n}^{-1}} k_{\lambda}\left(u_{1}, \ldots, u_{n-1}, 1\right) \prod_{j=1}^{n-1} u_{j}^{\lambda_{j}-\frac{\varepsilon}{p_{j}}-1} d u_{n-1} d u_{1} \cdots d u_{n-2}\right] d x_{n} \\
& =\int_{0}^{1} \int_{\mathbf{R}_{+}^{n-2}} k_{\lambda}\left(u_{1}, \ldots, u_{n-1}, 1\right) \prod_{j=1}^{n-1} u_{j}^{\lambda_{j}-\frac{\varepsilon}{p_{j}}-1}\left(\int_{1}^{u_{n-1}^{-1}} x_{n}^{-1} d x_{n}\right) d u_{1} \cdots d u_{n-1} \\
& =\int_{0}^{1} \int_{\mathbf{R}_{+}^{n-2}} k_{\lambda}\left(u_{1}, \ldots, u_{n-1}, 1\right) \prod_{j=1}^{n-1} u_{j}^{\lambda_{j}-\frac{\varepsilon}{p_{j}}-1}\left(-\ln u_{n-1}\right) d u_{1} \cdots d u_{n-1} \\
& \leq M \int_{0}^{1} \int_{\mathbf{R}_{+}^{n-2}} k_{\lambda}\left(u_{1}, \ldots, u_{n-1}, 1\right) \prod_{j=1}^{n-2} u_{j}^{\lambda_{j}-\frac{\varepsilon}{p_{j}}-1} u_{n-1}^{\lambda_{n-1}-\left(\frac{\varepsilon}{p_{n-1}}+\alpha\right)-1} d u_{1} \cdots d u_{n-1} \\
& \leq M \int_{\mathbf{R}_{+}^{n-1}} k_{\lambda}\left(u_{1}, \ldots, u_{n-1}, 1\right) \prod_{j=1}^{n-2} u_{j}^{\lambda_{j}-\frac{\varepsilon}{p_{j}}-1} u_{n-1}^{\lambda_{n-1}-\left(\frac{\varepsilon}{p_{n-1}}+\alpha\right)-1} d u_{1} \cdots d u_{n-1} \\
& =M \cdot k\left(\lambda_{1}-\frac{\varepsilon}{p_{1}}, \ldots, \lambda_{n-2}-\frac{\varepsilon}{p_{n-2}}, \lambda_{n-1}-\left(\frac{\varepsilon}{p_{n-1}}+\alpha\right)\right)<\infty .
\end{aligned}
$$


Hence by (10), we have

$$
I_{\varepsilon} \geq \int_{\mathbf{R}_{+}^{n-1}} k_{\lambda}\left(u_{1}, \ldots, u_{n-1}, 1\right) \prod_{j=1}^{n-1} u_{j}^{\lambda_{j}-\frac{\varepsilon}{p_{j}}-1} d u_{1} \cdots d u_{n-1}-o_{1}(1)
$$

Since by Lemma 3 we find

$$
\begin{aligned}
I_{\varepsilon} & \leq \varepsilon \int_{1}^{\infty} x_{n}^{-1-\varepsilon}\left[\int_{0}^{\infty} \cdots \int_{0}^{\infty} k_{\lambda}\left(u_{1}, \ldots, u_{n-1}, 1\right) \prod_{j=1}^{n-1} u_{j}^{\lambda_{j}-\frac{\varepsilon}{p_{j}}-1} d u_{1} \cdots d u_{n-1}\right] d x_{n} \\
& =\int_{0}^{\infty} \cdots \int_{0}^{\infty} k_{\lambda}\left(u_{1}, \ldots, u_{n-1}, 1\right) \prod_{j=1}^{n-1} u_{j}^{\lambda_{j}-\frac{\varepsilon}{p_{j}}-1} d u_{1} \cdots d u_{n-1} \\
& =k\left(\lambda_{1}-\frac{\varepsilon}{p_{1}}, \ldots, \lambda_{n-1}-\frac{\varepsilon}{p_{n-1}}\right)=k_{\lambda}+o_{2}(1),
\end{aligned}
$$

then combining with (11), we have (8).

Lemma 5 Suppose that $n \in \mathbf{N} \backslash\{1\}, p_{i} \in \mathbf{R} \backslash\{0,1\}(i=1, \ldots, n), \sum_{i=1}^{n} \frac{1}{p_{i}}=1, \frac{1}{q_{n}}=1-\frac{1}{p_{n}}$, $\left(\lambda_{1}, \ldots, \lambda_{n}\right) \in \mathbf{R}^{n}, \lambda_{n}=\sum_{i=1}^{n-1} \lambda_{i}=\frac{\lambda}{2}, k_{\lambda}\left(x_{1}, \ldots, x_{n}\right)(\geq 0)$ is a measurable function of $-\lambda-$ degree in $\mathbf{R}_{+}^{n}$ such that

$$
k_{\lambda}=\int_{\mathbf{R}_{+}^{n-1}} k_{\lambda}\left(u_{1}, \ldots, u_{n-1}, 1\right) \prod_{j=1}^{n-1} u_{j}^{\lambda_{j}-1} d u_{1} \cdots d u_{n-1} \in \mathbf{R} .
$$

If $f_{i} \geq 0$ are measurable functions in $\mathbf{R}_{+}(i=1, \ldots, n-1), \widetilde{k}_{\lambda}\left(x_{1}, \ldots, x_{n}\right):=k_{\lambda}\left(x_{1} x_{n}, \ldots, x_{n-1} x_{n}\right.$, 1), then (1) for $p_{i}>1(i=1, \ldots, n)$, we have

$$
\begin{aligned}
J & :=\left\{\int_{0}^{\infty} x_{n}^{\frac{\lambda q_{n}}{2}-1}\left[\int_{\mathbf{R}_{+}^{n-1}} \widetilde{k}_{\lambda}\left(x_{1}, \ldots, x_{n}\right) \prod_{i=1}^{n-1} f_{i}\left(x_{i}\right) d x_{1} \cdots d x_{n-1}\right]^{q_{n}} d x_{n}\right\}^{\frac{1}{q_{n}}} \\
& \leq k_{\lambda} \prod_{i=1}^{n-1}\left\{\int_{0}^{\infty} x^{p_{i}\left(1-\lambda_{i}\right)-1} f^{p_{i}}(x) d x\right\}^{\frac{1}{p_{i}}}
\end{aligned}
$$

(2) for $0<p_{1}<1, p_{i}<0(i=2, \ldots, n)$, we have the reverse of $(12)$.

Proof (1) For $p_{i}>1(i=1, \ldots, n)$, by the Hölder inequality (cf. [18]) and (7), it follows

$$
\begin{aligned}
& {\left[\int_{\mathbf{R}_{+}^{n-1}} \tilde{k}_{\lambda}\left(x_{1}, \ldots, x_{n}\right) \prod_{i=1}^{n-1} f_{i}\left(x_{i}\right) d x_{1} \cdots d x_{n-1}\right]^{q_{n}}} \\
& \quad=\left\{\int_{\mathbf{R}_{+}^{n-1}} \widetilde{k}_{\lambda}\left(x_{1}, \ldots, x_{n}\right) \prod_{i=1}^{n-1}\left[x_{i}^{\left(\lambda_{i}-1\right)\left(1-p_{i}\right)} \prod_{j=1(j \neq i)}^{n} x_{j}^{\lambda_{j}-1}\right]^{\frac{1}{p_{i}}} f_{i}\left(x_{i}\right)\right. \\
& \left.\quad \times\left[x_{n}^{\left(\lambda_{n}-1\right)\left(1-p_{n}\right)} \prod_{j=1}^{n-1} x_{j}^{\lambda_{j}-1}\right]^{\frac{1}{p_{n}}} d x_{1} \cdots d x_{n-1}\right\}^{q_{n}}
\end{aligned}
$$




$$
\begin{aligned}
\leq & \int_{\mathbf{R}_{+}^{n-1}} \widetilde{k}_{\lambda}\left(x_{1}, \ldots, x_{n}\right) \prod_{i=1}^{n-1}\left[x_{i}^{\left(\lambda_{i}-1\right)\left(1-p_{i}\right)} \prod_{j=1(j \neq i)}^{n} x_{j}^{\lambda_{j}-1}\right]^{\frac{q_{n}}{p_{i}}} f_{i}^{q_{n}}\left(x_{i}\right) d x_{1} \cdots d x_{n-1} \\
& \times\left\{\int_{\mathbf{R}_{+}^{n-1}} \widetilde{k}_{\lambda}\left(x_{1}, \ldots, x_{n}\right) x_{n}^{\left(\lambda_{n}-1\right)\left(1-p_{n}\right)} \prod_{j=1}^{n-1} x_{j}^{\lambda_{j}-1} d x_{1} \cdots d x_{n-1}\right\}^{q_{n}-1} \\
= & \left(k_{\lambda}\right)^{q_{n}-1} x_{n}^{1-q_{n} \lambda_{n}} \int_{\mathbf{R}_{+}^{n-1}} \widetilde{k}_{\lambda}\left(x_{1}, \ldots, x_{n}\right) \\
& \times \prod_{i=1}^{n-1}\left[x_{i}^{\left(\lambda_{i}-1\right)\left(1-p_{i}\right)} \prod_{j=1(j \neq i)}^{n} x_{j}^{\lambda_{j}-1}\right]^{\frac{q_{n}}{p_{i}}} f_{i}^{q_{n}}\left(x_{i}\right) d x_{1} \cdots d x_{n-1}, \\
J \leq & \left(k_{\lambda}\right)^{\frac{1}{p_{n}}}\left\{\int_{0}^{\infty} \int_{\mathbf{R}_{+}^{n-1}} \widetilde{k}_{\lambda}\left(x_{1}, \ldots, x_{n}\right)\right. \\
& \left.\times \prod_{i=1}^{n-1}\left[x_{i}^{\left(\lambda_{i}-1\right)\left(1-p_{i}\right)} \prod_{j=1(j \neq i)}^{n} x_{j}^{\lambda_{j}-1}\right]^{\frac{q_{n}}{p_{i}}} f_{i}^{q_{n}}\left(x_{i}\right) d x_{1} \cdots d x_{n-1} d x_{n}\right\}^{\frac{1}{q_{n}}} \\
= & \left(k_{\lambda}\right)^{\frac{1}{p_{n}}}\left\{\int_{\mathbf{R}_{+}^{n-1}}\left(\int_{0}^{\infty} \widetilde{k}_{\lambda}\left(x_{1}, \ldots, x_{n}\right) x_{n}^{\lambda_{n}-1} d x_{n}\right)\right. \\
& \left.\times \prod_{i=1}^{n-1}\left[x_{i}^{\left(\lambda_{i}-1\right)\left(1-p_{i}\right)} \prod_{j=1(j \neq i)}^{n-1} x_{j}^{\lambda_{j}-1}\right]^{\frac{q_{n}}{p_{i}}} f_{i}^{q_{n}}\left(x_{i}\right) d x_{1} \cdots d x_{n-1}\right\}^{\frac{1}{q_{n}}} .
\end{aligned}
$$

For $n \geq 3$, by the Hölder inequality again, it follows

$$
\begin{aligned}
J \leq & \left(k_{\lambda}\right)^{\frac{1}{p_{n}}}\left\{\prod _ { i = 1 } ^ { n - 1 } \left[\int_{\mathbf{R}_{+}^{n-1}}\left(\int_{0}^{\infty} \widetilde{k}_{\lambda}\left(x_{1}, \ldots, x_{n}\right) x_{n}^{\lambda_{n}-1} d x_{n}\right)\right.\right. \\
& \left.\left.\times x_{i}^{\left(\lambda_{i}-1\right)\left(1-p_{i}\right)} \prod_{j=1(j \neq i)}^{n-1} x_{j}^{\lambda_{j}-1} f_{i}^{p_{i}}\left(x_{i}\right) d x_{1} \cdots d x_{n-1}\right]^{\frac{q_{n}}{p_{i}}}\right\}^{\frac{1}{q_{n}}} \\
= & \left(k_{\lambda}\right)^{\frac{1}{p_{n}}} \prod_{i=1}^{n-1}\left\{\int _ { 0 } ^ { \infty } \left[\int_{\mathbf{R}_{+}^{n-1}} \widetilde{k}_{\lambda}\left(x_{1}, \ldots, x_{n}\right)\right.\right. \\
& \left.\left.\times x_{i}^{\lambda_{i}} \prod_{j=1(j \neq i)}^{n} x_{j}^{\lambda_{j}-1} d x_{1} \cdots d x_{i-1} d x_{i+1} \cdots d x_{n}\right] x_{i}^{p_{i}\left(1-\lambda_{i}\right)-1} f_{i}^{p_{i}}\left(x_{i}\right) d x_{i}\right\}^{\frac{1}{p_{i}}} \\
= & \left(k_{\lambda}\right)^{\frac{1}{p_{n}}} \prod_{i=1}^{n-1}\left\{\int_{0}^{\infty} \omega_{i}\left(x_{i}\right) x_{i}^{p_{i}\left(1-\lambda_{i}\right)-1} f_{i}^{p_{i}}\left(x_{i}\right) d x_{i}\right\}^{\frac{1}{p_{i}}} .
\end{aligned}
$$

Then by (7), we have (12). (Note: for $n=2$, we do not use the Hölder inequality again in the above.) (2) For $0<p_{1}<1, p_{i}<0(i=2, \ldots, n)$, by the reverse Hölder inequality and in the same way, we obtain the reverses of (12).

\section{Main results and applications}

With the assumptions given in Lemma 5 , setting $\phi_{i}(x):=x^{p_{i}\left(1-\lambda_{i}\right)-1}(x \in(0, \infty) ; i=1, \ldots, n)$, then we find $\phi_{n}^{1 /\left(1-p_{n}\right)}(x)=x^{q_{n} \lambda_{n-1}}$. If $p_{i}>1(i=1, \ldots, n)$, define the following real function 
spaces:

$$
\begin{aligned}
& L_{\phi_{i}}^{p_{i}}(0, \infty):=\left\{f ;\|f\|_{p_{i} \phi_{i}}=\left\{\int_{0}^{\infty} \phi_{i}(x)|f(x)|^{p_{i}} d x\right\}^{\frac{1}{p_{i}}}<\infty\right\} \quad(i=1, \ldots, n), \\
& \prod_{i=1}^{n-1} L_{\phi_{i}}^{p_{i}}(0, \infty):=\left\{\left(f_{1}, \ldots, f_{n-1}\right) ; f_{i} \in L_{\phi_{i}}^{p_{i}}(0, \infty), i=1, \ldots, n-1\right\},
\end{aligned}
$$

and a multiple Hilbert-type integral operator $T: \prod_{i=1}^{n-1} L_{\phi_{i}}^{p_{i}}(0, \infty) \rightarrow L_{\phi_{n}^{1 /\left(1-p_{n}\right)}}^{q_{n}}$ as follows: for $f=\left(f_{1}, \ldots, f_{n-1}\right) \in \prod_{i=1}^{n-1} L_{\phi_{i}}^{p_{i}}(0, \infty)$,

$$
(T f)\left(x_{n}\right):=\int_{\mathbf{R}_{+}^{n-1}} \widetilde{k}_{\lambda}\left(x_{1}, \ldots, x_{n}\right) \prod_{i=1}^{n-1} f_{i}\left(x_{i}\right) d x_{1} \cdots d x_{n-1}, \quad x_{n} \in(0, \infty) .
$$

Then by (12), it follows $T f \in L_{\phi_{n}^{1 /\left(1-p_{n}\right)}}^{q_{n}}, T$ is bounded, $\|T f\|_{q_{n}, \phi_{n}^{1 /\left(1-p_{n}\right)}} \leq k_{\lambda} \prod_{i=1}^{n-1}\left\|f_{i}\right\|_{p_{i}, \phi_{i}}$ and $\|T\| \leq k_{\lambda}$, where

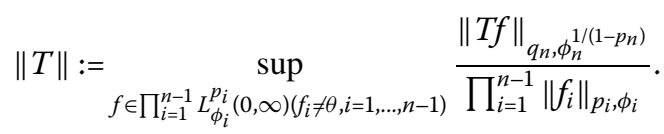

Define the formal inner product of $T\left(f_{1}, \ldots, f_{n-1}\right)$ and $f_{n}$ as

$$
\left(T\left(f_{1}, \ldots, f_{n-1}\right), f_{n}\right):=\int_{\mathbf{R}_{+}^{n}} \widetilde{k}_{\lambda}\left(x_{1}, \ldots, x_{n}\right) \prod_{i=1}^{n} f_{i}\left(x_{i}\right) d x_{1} \cdots d x_{n} .
$$

Theorem 1 With the assumptions given in Lemma 5 , suppose that for any $\left(\lambda_{1}, \ldots, \lambda_{n}\right) \in \mathbf{R}^{n}$, it satisfies $\lambda_{n}=\sum_{i=1}^{n-1} \lambda_{i}=\frac{\lambda}{2}$, and

$$
0<k_{\lambda}=\int_{\mathbf{R}_{+}^{n-1}} k_{\lambda}\left(u_{1}, \ldots, u_{n-1}, 1\right) \prod_{j=1}^{n-1} u_{j}^{\lambda_{j}-1} d u_{1} \cdots d u_{n-1}<\infty .
$$

If $f_{i}(\geq 0) \in L_{\phi_{i}}^{p_{i}}(0, \infty),\|f\|_{p_{i, \phi_{i}}}>0(i=1, \ldots, n)$, then (i) for $p_{i}>1(i=1, \ldots, n)$, we have $\|T\|=$ $k_{\lambda}$ and the following equivalent inequalities:

$$
\begin{aligned}
& \left\|T\left(f_{1}, \ldots, f_{n-1}\right)\right\|_{q_{n}, \phi_{n}^{1 /\left(1-p_{n}\right)}}<k_{\lambda} \prod_{i=1}^{n-1}\left\|f_{i}\right\|_{p_{i}, \phi_{i}}, \\
& \left(T\left(f_{1}, \ldots, f_{n-1}\right), f_{n}\right)<k_{\lambda} \prod_{i=1}^{n}\left\|f_{i}\right\|_{p_{i}, \phi_{i}},
\end{aligned}
$$

where the constant factor $k_{\lambda}$ is the best possible; (ii) for $0<p_{1}<1, p_{i}<0(i=2, \ldots, n)$, using the formal symbols in the case of (i), we have the equivalent reverses of (19) and (20) with the same best constant factor. 
Proof (i) For all $p_{i}>1$, if (12) takes the form of equality, then for $n \geq 3$ in (14), there exist $C_{i}$ and $C_{k}(i \neq k)$ such that they are not all zero and

$$
\begin{aligned}
& C_{i} x_{i}^{\left(\lambda_{i}-1\right)\left(1-p_{i}\right)} \prod_{j=1(j \neq i)}^{n-1} x_{j}^{\lambda_{j}-1} f_{j}^{p_{j}}\left(x_{j}\right) \\
& =C_{k} x_{k}^{\left(\lambda_{k}-1\right)\left(1-p_{k}\right)} \prod_{j=1(j \neq k)}^{n-1} x_{j}^{\lambda_{j}-1} f_{j}^{p_{j}}\left(x_{j}\right) \text { a.e. in } \mathbf{R}_{+}^{n},
\end{aligned}
$$

e.t. $C_{i} x_{i}^{p_{i}\left(1-\lambda_{i}\right)} f_{i}^{p_{i}}\left(x_{i}\right)=C_{k} x_{k}^{p_{k}\left(1-\lambda_{k}\right)} f_{k}^{p_{k}}\left(x_{k}\right)=C$ a.e. in $\mathbf{R}_{+}^{n}$. Assuming that $C_{i}>0$, then $x_{i}^{p_{i}\left(1-\lambda_{i}\right)-1} f_{i}^{p_{i}}\left(x_{i}\right)=C /\left(C_{i} x_{i}\right)$, which contradicts $\|f\|_{p_{i, \phi_{i}}}>0$. (Note: for $n=2$, we consider (13) for $f_{k}^{p_{i}}\left(x_{k}\right)=1$ in the above.) Hence we have (19). By the Hölder inequality, it follows

$$
\begin{aligned}
\left(T f, f_{n}\right) & =\int_{0}^{\infty}\left(x_{n}^{\lambda_{n}-\frac{1}{q_{n}}} \int_{\mathbf{R}_{+}^{n-1}} \widetilde{k}_{\lambda}\left(x_{1}, \ldots, x_{n}\right) \prod_{i=1}^{n-1} f_{i}\left(x_{i}\right) d x_{1} \cdots d x_{n-1}\right)\left(x_{n}^{\frac{1}{q_{n}}-\lambda_{n}} f_{n}\left(x_{n}\right)\right) d x_{n} \\
& \leq\left\|T\left(f_{1}, \ldots, f_{n-1}\right)\right\|_{q_{n}, \phi_{n}^{1 /\left(1-p_{n}\right)} \|}\left\|f_{n}\right\|_{p_{n}, \phi_{n}}
\end{aligned}
$$

and then by (19), we have (20). Assuming that (20) is valid, setting

$$
f_{n}\left(x_{n}\right):=x_{n}^{q_{n} \lambda_{n}-1}\left[\int_{\mathbf{R}_{+}^{n-1}} \widetilde{k}_{\lambda}\left(x_{1}, \ldots, x_{n}\right) \prod_{i=1}^{n-1} f_{i}\left(x_{i}\right) d x_{1} \cdots d x_{n-1}\right]^{q_{n}-1}
$$

then $J=\left\{\int_{0}^{\infty} x_{n}^{p_{n}\left(1-\lambda_{n}\right)-1} f_{n}^{p_{n}}\left(x_{n}\right) d x_{n}\right\}^{\frac{1}{q_{n}}}$. By (12), it follows $J<\infty$. If $J=0$, then (19) is naturally valid. Assuming that $0<J<\infty$, by (20), it follows

$$
\begin{aligned}
& \int_{0}^{\infty} x_{n}^{p_{n}\left(1-\lambda_{n}\right)-1} f_{n}^{p_{n}}\left(x_{n}\right) d x_{n}=J^{q_{n}}=\left(T f, f_{n}\right)<k_{\lambda} \prod_{i=1}^{n}\left\|f_{i}\right\|_{p_{i}, \phi_{i}}, \\
& \left\{\int_{0}^{\infty} x_{n}^{p_{n}\left(1-\lambda_{n}\right)-1} f_{n}^{p_{n}}\left(x_{n}\right) d x_{n}\right\}^{\frac{1}{q_{n}}}=J<k_{\lambda} \prod_{i=1}^{n-1}\left\|f_{i}\right\|_{p_{i}, \phi_{i}},
\end{aligned}
$$

and then (19) is valid, which is equivalent to (20).

For $\varepsilon>0$ small enough, setting $\widetilde{f}_{i}(x)$ as: $\widetilde{f}_{i}(x)=0, x \in(0,1) ; \widetilde{f}_{i}(x)=x^{\lambda_{i}-\frac{\varepsilon}{p_{i}}-1}, x \in[1, \infty)$ $(i=1, \ldots, n-1), \widetilde{f}_{n}(x)=x^{\lambda_{n}+\frac{\varepsilon}{p_{n}}-1}, x \in(0,1) ; \widetilde{f}_{n}(x)=0, x \in[1, \infty)$, if there exists $k \leq k_{\lambda}$ such that (20) is still valid as we replace $k_{\lambda}$ by $k$, then in particular, by Lemma 4 , we have

$$
k_{\lambda}+o(1)=I_{\varepsilon}=\varepsilon\left(T\left(\widetilde{f}_{1}, \ldots, \widetilde{f}_{n-1}\right), \widetilde{f}_{n}\right)<\varepsilon k \prod_{i=1}^{n}\left\|\widetilde{f}_{i}\right\|_{p_{i}, \phi_{i}}=k
$$

and $k_{\lambda} \leq k\left(\varepsilon \rightarrow 0^{+}\right)$. Hence $k=k_{\lambda}$ is the best value of (20). We confirm that the constant factor $k_{\lambda}$ in (19) is the best possible, otherwise we can get a contradiction by (21) that the constant factor in (20) is not the best possible. Therefore $\|T\|=k_{\lambda}$.

(ii) For $0<p_{1}<1, p_{i}<0(i=2, \ldots, n)$, by using the reverse Hölder inequality and in the same way, we have the equivalent reverses of (19) and (20) with the same best constant factor. 
Example 1 For $\lambda>0, \lambda_{i}=\frac{\lambda}{r_{i}}(i=1, \ldots, n), r_{n}=2, \sum_{i=1}^{n} \frac{1}{r_{i}}=1, k_{\lambda}\left(x_{1}, \ldots, x_{n}\right)=\frac{1}{\left(\sum_{i=1}^{n} x_{i}\right)^{\lambda}}$, by mathematical induction, we can show

$$
k_{\lambda}=\int_{R_{+}^{n-1}} \frac{1}{\left(\sum_{i=1}^{n-1} u_{i}+1\right)^{\lambda}} \prod_{j=1}^{n-1} u_{j}^{\frac{\lambda}{r_{j}}-1} d u_{1} \cdots d u_{n-1}=\frac{1}{\Gamma(\lambda)} \prod_{i=1}^{n} \Gamma\left(\frac{\lambda}{r_{i}}\right) .
$$

In fact, for $n=2$, we obtain

$$
k_{\lambda}=\int_{R_{+}} \frac{1}{\left(u_{1}+1\right)^{\lambda}} u_{1}^{\frac{\lambda}{r_{1}}-1} d u_{1}=\frac{1}{\Gamma(\lambda)} \Gamma\left(\frac{\lambda}{r_{1}}\right) \Gamma\left(\frac{\lambda}{r_{2}}\right) .
$$

Assuming that for $n(\geq 2)(22)$ is valid, then for $n+1$, it follows

$$
\begin{aligned}
k_{\lambda} & =\int_{R_{+}^{n}} \frac{1}{\left(\sum_{i=1}^{n} u_{i}+1\right)^{\lambda}} \prod_{j=1}^{n} u_{j}^{\frac{\lambda}{r_{j}}-1} d u_{1} \cdots d u_{n} \\
& =\int_{R_{+}^{n-1}} \prod_{j=2}^{n} u_{j}^{\frac{\lambda}{r_{j}}-1}\left[\int_{R_{+}} \frac{1}{\left[u_{1}+\left(\sum_{i=2}^{n} u_{i}+1\right)\right]^{\lambda}} u_{1}^{\frac{\lambda}{r_{1}}-1} d u_{1}\right] d u_{2} \cdots d u_{n} \\
& =\int_{R_{+}^{n-1}} \frac{1}{\left(\sum_{i=2}^{n} u_{i}+1\right)^{\lambda}} \prod_{j=2}^{n} u_{j}^{\frac{\lambda}{r_{j}}-1}\left[\int_{R_{+}} \frac{1}{\left(v_{1}+1\right)^{\lambda}} v_{1}^{\frac{\lambda}{r_{1}}-1} d v_{1}\right] d u_{2} \cdots d u_{n} \\
& =\frac{\Gamma\left(\frac{\lambda}{r_{1}}\right) \Gamma\left(\lambda-\frac{\lambda}{r_{1}}\right)}{\Gamma(\lambda)} \int_{R_{+}^{n-1}} \frac{1}{\left(\sum_{i=2}^{n} u_{i}+1\right)^{\lambda\left(1-\frac{1}{r_{1}}\right)}} \prod_{j=2}^{n} u_{j}^{\frac{\lambda}{r_{j}}-1} d u_{2} \cdots d u_{n} \\
& =\frac{\Gamma\left(\frac{\lambda}{r_{1}}\right) \Gamma\left(\lambda-\frac{\lambda}{r_{1}}\right)}{\Gamma(\lambda)} \frac{1}{\Gamma\left(\lambda-\frac{\lambda}{r_{1}}\right)} \prod_{i=2}^{n+1} \Gamma\left(\frac{\lambda}{r_{i}}\right)=\frac{1}{\Gamma(\lambda)} \prod_{i=1}^{n+1} \Gamma\left(\frac{\lambda}{r_{i}}\right) .
\end{aligned}
$$

Then by mathematical induction, (22) is valid for $n \in \mathbf{N} \backslash\{1\}$.

Example 2 For $\lambda>0, \lambda_{i}=\frac{\lambda}{r_{i}}(i=1, \ldots, n), r_{n}=2, \sum_{i=1}^{n} \frac{1}{r_{i}}=1, k_{\lambda}\left(x_{1}, \ldots, x_{n}\right)=\frac{1}{\left(\max _{1 \leq i \leq n}\left\{x_{i}\right)\right)^{\lambda}}$, by mathematical induction, we can show

$$
k_{\lambda}=\int_{R_{+}^{n-1}} \frac{1}{\left(\max _{1 \leq i \leq n-1}\left\{u_{i}\right\}+1\right)^{\lambda}} \prod_{j=1}^{n-1} u_{j}^{\frac{\lambda}{r_{j}}-1} d u_{1} \cdots d u_{n-1}=\frac{\prod_{i=1}^{n} r_{i}}{\lambda^{n-1}} .
$$

In fact, for $n=2$, we obtain

$$
k_{\lambda}=\int_{R_{+}} \frac{u_{1}^{\frac{\lambda}{r_{1}}}-1}{\left(\max \left\{u_{1}, 1\right\}\right)^{\lambda}} d u_{1}=\int_{0}^{1} u_{1}^{\frac{\lambda}{r_{1}}-1} d u_{1}+\int_{1}^{\infty} u_{1}^{\frac{-\lambda}{r_{2}}-1} d u_{1}=\frac{1}{\lambda} r_{1} r_{2}
$$

Assuming that for $n(\geq 2)$, (22) is valid, then for $n+1$, it follows

$$
\begin{aligned}
k_{\lambda} & =\int_{R_{+}^{n-1}} \prod_{j=2}^{n} u_{j}^{\frac{\lambda}{r_{j}}-1}\left[\int_{0}^{\infty} \frac{1}{\left(\max _{1 \leq i \leq n}\left\{u_{i}, 1\right\}\right)^{\lambda}} u_{1}^{\frac{\lambda}{r_{1}}-1} d u_{1}\right] d u_{2} \cdots d u_{n} \\
& =\int_{R_{+}^{n-1}} \prod_{j=2}^{n} u_{j}^{\frac{\lambda}{r_{j}}-1}\left[\int_{0}^{\max \left\{u_{2}, \ldots, u_{n}, 1\right\}} \frac{1}{\left(\max _{2 \leq i \leq n}\left\{u_{i}, 1\right\}\right)^{\lambda}} u_{1}^{\frac{\lambda}{r_{1}}-1} d u_{1}\right.
\end{aligned}
$$


Huang and Yang Journal of Inequalities and Applications 2013, 2013:73

Page 11 of 12

http://www.journalofinequalitiesandapplications.com/content/2013/1/73

$$
\begin{aligned}
& \left.+\int_{\max \left\{u_{2}, \ldots, u_{n}, 1\right\}}^{\infty} \frac{1}{u_{1}^{\lambda}} u_{1}^{\frac{\lambda}{r_{1}}-1} d u_{1}\right] d u_{2} \cdots d u_{n} \\
= & \frac{r_{1}^{2}}{\lambda\left(r_{1}-1\right)} \int_{R_{+}^{n-1}} \frac{1}{\left(\max _{2 \leq i \leq n}\left\{u_{i}, 1\right\}\right)^{\lambda\left(1-\frac{1}{r_{1}}\right)}} \prod_{j=2}^{n} u_{j}^{\frac{\lambda}{r_{j}}-1} d u_{2} \cdots d u_{n} \\
= & \frac{r_{1}^{2}}{\lambda\left(r_{1}-1\right)}\left(\frac{r_{1}}{r_{1}-1}\right)^{n-1} \int_{R_{+}^{n-1}} \frac{1}{\left(\max _{2 \leq i \leq n}\left\{v_{i}, 1\right\}\right)^{\lambda}} \prod_{j=2}^{n} v_{j}^{\frac{\lambda}{r_{j}} \frac{r_{1}}{r_{1}-1}-1} d v_{2} \cdots d v_{n} \\
= & \frac{r_{1}^{2}}{\lambda\left(r_{1}-1\right)}\left(\frac{r_{1}}{r_{1}-1}\right)^{n-1} \frac{1}{\lambda^{n-1}} \prod_{i=2}^{n+1} \frac{r_{1}-1}{r_{1}} r_{i}=\frac{1}{\lambda^{n}} \prod_{i=1}^{n+1} r_{i} .
\end{aligned}
$$

Then by mathematical induction, (23) is valid for $n \in \mathbf{N} \backslash\{1\}$.

Example 3 For $\lambda>0, \lambda_{i}=\frac{-\lambda}{r_{i}}(i=1, \ldots, n), r_{n}=2, \sum_{i=1}^{n} \frac{1}{r_{i}}=1, \quad k_{\lambda}\left(x_{1}, \ldots, x_{n}\right)=$ $\left(\min _{1 \leq i \leq n}\left\{x_{i}\right\}\right)^{\lambda}$, by mathematical induction, we can show

$$
k_{-\lambda}=\int_{R_{+}^{n-1}}\left(\min \left\{u_{1}, \ldots, u_{n-1}, 1\right\}\right)^{\lambda} \prod_{j=1}^{n-1} u_{j}^{\frac{-\lambda}{r_{j}}-1} d u_{1} \cdots d u_{n-1}=\frac{\prod_{i=1}^{n} r_{i}}{\lambda^{n-1}}
$$

In fact, for $n=2$, we obtain

$$
k_{-\lambda}=\int_{0}^{1} u_{1}^{\frac{\lambda}{r_{2}}-1} d u_{1}+\int_{1}^{\infty} u_{1}^{\frac{-\lambda}{r_{1}}-1} d u_{1}=\frac{1}{\lambda} r_{1} r_{2}
$$

Assuming that for $n(\geq 2),(24)$ is valid, then for $n+1$, it follows

$$
\begin{aligned}
k_{-\lambda}= & \int_{R_{+}^{n-1}} \prod_{j=2}^{n} u_{j}^{\frac{-\lambda}{r_{j}}-1}\left[\int_{0}^{\infty}\left(\min \left\{u_{1}, \ldots, u_{n}, 1\right\}\right)^{\lambda} u_{1}^{\frac{-\lambda}{r_{1}}-1} d u_{1}\right] d u_{2} \cdots d u_{n} \\
= & \int_{R_{+}^{n-1}} \prod_{j=2}^{n} u_{j}^{\frac{-\lambda}{r_{j}}-1}\left[\int_{0}^{\min \left\{u_{2}, \ldots, u_{n}, 1\right\}} u_{1}^{\lambda} u_{1}^{\frac{-\lambda}{r_{1}}-1} d u_{1}\right. \\
& \left.+\int_{\min \left\{u_{2}, \ldots, u_{n}, 1\right\}}^{\infty}\left(\min \left\{u_{2}, \ldots, u_{n}, 1\right\}\right)^{\lambda} u_{1}^{\frac{-\lambda}{r_{1}}-1} d u_{1}\right] d u_{2} \cdots d u_{n} \\
= & \frac{r_{1}^{2}}{\lambda\left(r_{1}-1\right)} \int_{R_{+}^{n-1}}\left(\min \left\{u_{2}, \ldots, u_{n}, 1\right\}\right)^{\lambda\left(1-\frac{1}{r_{1}}\right)} \prod_{j=2}^{n} u_{j}^{\frac{-\lambda\left(1-\frac{1}{r_{1}}\right)}{\left(1-\frac{1}{r_{1}}\right) r_{j}}-1} d u_{2} \cdots d u_{n} \\
= & \frac{r_{1}^{2}}{\lambda\left(r_{1}-1\right)} \frac{1}{\left[\lambda\left(1-\frac{1}{r_{1}}\right)\right]^{n-1}} \prod_{i=2}^{n+1}\left(1-\frac{1}{r_{1}}\right) r_{i}=\frac{1}{\lambda^{n}} \prod_{i=1}^{n+1} r_{i} .
\end{aligned}
$$

Then, by mathematical induction, (24) is valid for $n \in \mathbf{N} \backslash\{1\}$.

Remarks (i) In particular, for $n=2$ in (20), we have

$$
\begin{aligned}
& \int_{0}^{\infty} \int_{0}^{\infty} k_{\lambda}(1, x y) f(x) g(y) d x d y \\
& \quad<k_{\lambda}\left\{\int_{0}^{\infty} x^{p\left(1-\frac{\lambda}{2}\right)-1} f^{p}(x) d x\right\}^{\frac{1}{p}}\left\{\int_{0}^{\infty} x^{q\left(1-\frac{\lambda}{2}\right)-1} g^{q}(x) d x\right\}^{\frac{1}{q}},
\end{aligned}
$$


where $k_{\lambda}=\int_{0}^{\infty} k_{\lambda}(u, 1) u^{\frac{\lambda}{2}-1} d u>0(\lambda \in \mathbf{R})$ is the best possible. Inequality (25) is an extension of (4) and (8.1.7) in [8].

(ii) In Examples 1 and 2, by Theorem 1, since for any $\left(\lambda_{1}, \ldots, \lambda_{n}\right) \in \mathbf{R}^{n}\left(\lambda_{n}=\sum_{i=1}^{n} \lambda_{i}=\frac{\lambda}{2}\right)$, we obtain $0<k_{\lambda}<\infty$, then we have $\|T\|=k_{\lambda}$ and the equivalent inequalities (19) and (20) with the particular kernels and some equivalent reverses. In Example 3, still using Theorem 1, we find $0<\|T\|=k_{-\lambda}<\infty$ and the relating particular inequalities.

\section{Competing interests}

The authors declare that they have no competing interests.

\section{Authors' contributions}

QH carried out the study, and wrote the manuscript. BY participated in the design of the study, and reformed the manuscript. All authors read and approved the final manuscript.

\section{Acknowledgements}

This work is supported by Guangdong Modern Information Service industry Develop Particularly item 2011 (No. 13090 ).

Received: 3 October 2012 Accepted: 11 February 2013 Published: 28 February 2013

\section{References}

1. Hardy, GH, Littlewood, JE, Pólya, G: Inequalities. Cambridge University Press, Cambridge (1934)

2. Mitrinović, DS, Pečarić, JE, Fink, AM: Inequalities Involving Functions and Their Integrals and Derivatives. Kluwer Academic, Boston (1991)

3. Bicheng, Y: A survey of the study of Hilbert-type inequalities with parameters. Adv. Math. 28(3), 257-268 (2009)

4. Zhang, K: A bilinear inequality. J. Math. Anal. Appl. 271, 288-296 (2002)

5. Yang, B: On a extension of Hilbert's integral inequality with some parameters. Aust. J. Math. Anal. Appl. 1(1), Art. 11 (2004)

6. Yang, B: A new Hilbert's type integral inequality. Soochow J. Math. 33(4), 849-859 (2007)

7. Yang, B: A Hilbert-type integral inequality with a non-homogeneous kernel. Journal of Xiamen University (Natural Science) 48(2), 165-169 (2009)

8. Yang, B: The Norm of Operator and Hilbert-Type Inequalities. Science Press, Beijing (2009) (China)

9. Hong, Y: All-side generalization about Hardy-Hilbert integral inequalities. Acta Math. Sin., 44(4), 619-625 (2001)

10. He, L, Yu, J, Gao, M: An extension of Hilbert's integral inequality. Journal of Shaoguan University (Natural Science), 23(3), 25-30 (2002)

11. Yang, B: On a multiple Hardy-Hilbert's integral inequality. Chin. Ann. Math. 24A(6), 25-30 (2003)

12. Yang, B, Rassias, T: On the way of weight coefficient and research for Hilbert-type inequalities. Math. Inequal. Appl. 6(4), 625-658 (2003)

13. Yang, B, Brnetić, I, Krnić, M, Pećarić, J: Generalization of Hilbert and Hardy-Hilbert integral inequalities. Math. Inequal. Appl. 8(2), 259-272 (2005)

14. Bicheng, Y: On the norm of an integral operator and applications. J. Math. Anal. Appl. 321, 182-192 (2006)

15. Bicheng, $Y$ : On the norm of a self-adjoint operator and a new bilinear integral inequality. Acta Math. Sin. Engl. Ser. 23(7), 1311-1316 (2007)

16. Benyi, A, Choonghong, O: Best constant for certain multilinear integral operator. J. Inequal. Appl. 2006, Art. ID. 28582 (2006)

17. Kuang, J: Introduction to Real Analysis. Hunan Education Press, Chansha (1996) (China)

18. Kuang, J: Applied Inequalities. Shangdong Science Technic Press, Jinan (2004) (China) 